\title{
Neutrophil to lymphocyte rate and serum prealbumin maybe predictors for abnormal high blood pressure caused by adrenocorticotropic hormone therapy in children with epileptic spasms: two cases report
}

\author{
Hao Li ${ }^{1,2,3 \#}$, Cui-Jin Wang ${ }^{1 \#}$, Yun-Qing Zhou ${ }^{1 \#}$, Ying-Yan Wang ${ }^{1}$, Chang-Hua Mou ${ }^{1}$, Shun-Guo Zhang ${ }^{3}$, \\ Ji-Wen Wang ${ }^{1}$ \\ ${ }^{1}$ Department of Neurology, Shanghai Children's Medical Center, Shanghai Jiao Tong University School of Medicine, Shanghai 200127, China; \\ ${ }^{2}$ Clinical Research Center, Shanghai Children's Medical Center, National Children's Medical Center, Shanghai Jiao Tong University School of \\ Medicine, Shanghai 200127, China; ${ }^{3}$ Department of Pharmacy, Shanghai Children's Medical Center, Shanghai Jiao Tong University School of \\ Medicine, Shanghai 200127, China \\ \#These authors contributed equally to this work. \\ Correspondence to: Shun-Guo Zhang. Department of Pharmacy, Shanghai Children's Medical Center, Shanghai Jiao Tong University School of \\ Medicine, Shanghai 200127, China. Email: zsguocn@sina.cn; Ji-Wen Wang, Department of Neurology, Shanghai Children's Medical Center, \\ Shanghai Jiao Tong University School of Medicine, Shanghai 200127, China. Email: wangjiwen@scmc.com.cn.
}

\begin{abstract}
Epileptic spasms are a catastrophic form of epilepsy. When epileptic spasms occur under 2-yearold, they may be also called "infantile spasms". Adrenocorticotropic hormone (ACTH) is recommended as first line intervention for the treatment of epileptic spasms without tuberous sclerosis complex. The chief risks of ACTH therapy are immunosuppression and hypertension. We reported rare cases of abnormal high blood pressure in two male epileptic spasms patients during ACTH therapy. Both patients' blood pressure reached a high blood pressure stage 2 on the 9th day and 10th day of ACTH treatment, respectively. The blood pressure returned to normal range after the drug dosage was reduced or stopped. The lower level of neutrophil\%, neutrophil count, and a higher level of lymphocyte $\%$, lymphocyte count and prealbumin than normal range were observed in both patients before ACTH therapy. The neutrophil to lymphocyte rate might be a predictor for high blood pressure among patients treated with ACTH. The rates of both patients were under 0.50 (0.42 for Case 1 and 0.17 for Case 2). We reported the documented cases in two Chinese pediatric patients who suffered from epileptic spasms treated with ACTH resulted in abnormal high blood pressure, which could be predicted by using neutrophil to lymphocyte rate. We also mentioned serum prealbumin might be another predictor. More clinical data is required to elucidate the relationship between serum prealbumin level and blood pressure.
\end{abstract}

Keywords: Adrenocorticotropic hormone (ACTH); neutrophil; lymphocyte; prealbumin; high blood pressure; epileptic spasms; infantile spasms

Submitted Nov 13, 2019. Accepted for publication Jan 10, 2020.

doi: $10.21037 /$ atm.2020.01.132

View this article at: http://dx.doi.org/10.21037/atm.2020.01.132

\section{Introduction}

Epileptic spasms are characterized by a sustained tonic contraction of the neck, trunk, brief flexion or extension of the arms and flexion at the waist which usually tend to occur in clusters (1-3). Both semiology of epileptic spasms and the interictal pattern of hypsarrhythmia on the electroencephalography (EEG) are important in the diagnosis of epileptic spasms (3). When epileptic 
spasms occur during the infantile stage of life, they may be also called "infantile spasms" (2). Epileptic spasms are a devastating form of epilepsy with the peak onset between 4 and 7 months of age, occurring in 0.25 and 0.42 per 1,000 live births per year $(2,4-6)$. Hypoxic-ischemic encephalopathy, cerebral malformations, cerebrovascular disease such as stroke, chromosomal, tuberous sclerosis complex, periventricular leukomalacia and hemorrhage are the most common etiologies for epileptic spasms (7). However, only $61 \%$ of epileptic spasms had a proven etiology, about one-third of epileptic spasms were cryptogenic (7). Children with epileptic spasms often have developmental disabilities (6).

Adrenocorticotropic hormone (ACTH), corticosteroids, vigabatrin and ketogenic diet are clinical interventions for epileptic spasms treatment, but the response rates of these methods are less than $60 \%$, and $18 \%$ of responders later relapsed (8-10). Among these treatment interventions, ACTH therapy is considered preferentially treatment for epileptic spasms with a response rate of $55 \%$ (8). There are two kinds of ACTH on the market worldwide, which are natural ACTH and synthetic ACTH. Natural ACTH is 39 amino acids in length, which is a highly purified sterile preparation of the ACTH extracted from the pituitary gland of animals, available in the USA and China (11). Synthetic ACTH is a truncated peptide spanning the first 24 residues of a natural ACTH with a therapeutic effect as the same as natural ACTH. Both natural ACTH and synthetic ACTH are used for the treatment of epileptic spasms (12). However, immunosuppression and hypertension are two chief risks for epileptic spasms patients treated with ACTH $(12,13)$. Hypertension, especially in infantile patients, may leads to congestive heart failure (12). Screening for asymptomatic hypertension and bio-markers for potential hypertension caused by ACTH therapy are key safety measures need to be done before ACTH therapy. We reported such rare cases of abnormal high blood pressure during ACTH therapy in two pediatric patients who presented with epileptic spasms. Both prealbumin and lymphocyte level of these two patients were higher than normal range, but neutrophil level was lower than normal range before ACTH therapy.

\section{Case presentation}

\section{Case 1}

The present patient was a 2-year-7-month-old Chinese boy suffering from epileptic spasms for about one year.
He was initially hospitalized in June 2018 for appearance of paroxysmal nods for three to four times per day and occasionally accompanied by limb shaking. The symptoms gradually worsened. Abnormal EEG was observed during video EEG in Children's Hospital of Soochow University (detailed description of EEG was presented in Table 1, oscillogram of EEG was not available). This patient was diagnosed with infantile spasms and treated with ACTH later (25 U, qd, intravenously guttae, for 28 days) in Soochow. After 28 days ACTH therapy, this patient was still accompanied with nodding until half a month treatment with topiramate and prednisone. In March 2019, the nodding attack appeared again and gradually increased up to more than 20 times for a cluster. The dosage of topiramate $(50 \mathrm{mg}, \mathrm{q} 12 \mathrm{~h})$ was gradually adjusted in the outpatient department, and nitrazepam tablet was added. The nodding attack was slightly improved later. This patient was hospitalized in our hospital on June $1^{\text {st }}, 2019$ for further epileptic spasms treatment.

Clinical characteristics of this patient before ACTH therapy were shown in Table 1. The blood pressure of this patient was 104/64 mmHg before ACTH therapy. Results of main laboratory test during ACTH therapy were listed in Table 2. The ACTH treatment cycle and dosage of this patient were shown in Figure 1. After ACTH treatment, the blood pressure increased to an abnormal range on the $9^{\text {th }}$ day $(117 / 78 \mathrm{mmHg})$. After adjusting the ACTH therapy dose from $50 \mathrm{U}$ to $25 \mathrm{U}$, the patient completed the ACTH therapy for 14 days, but the blood pressure of this patient reached a maximum of $119 / 62 \mathrm{mmHg}$ on the first day after ACTH treatment (Figure 1A). The blood pressure of the patient returned to normal 4 days later. There was no abnormal increase in blood pressure of this patient after discharge from hospital.

\section{Case 2}

The present patient was a 6-month-old Chinese boy suffering from epileptic spasms for more than one month. He began to have spasms attack since May 2019. The clinical symptoms were nodding, rolling eyes and flexion of limbs for three times a day, 5 to 6 clusters each time. There was no obvious rigidity shaking, unconsciousness loss, lip cyanosis and abnormal complexion during the seizures. Before hospitalized, results from the general EEG in the outpatient department showed epileptiform activity in both occipital and temporal areas (Figure 2). Considering this patient was suffered from epileptic spasms and ACTH 
Table 1 Clinical baseline characteristics of two cases

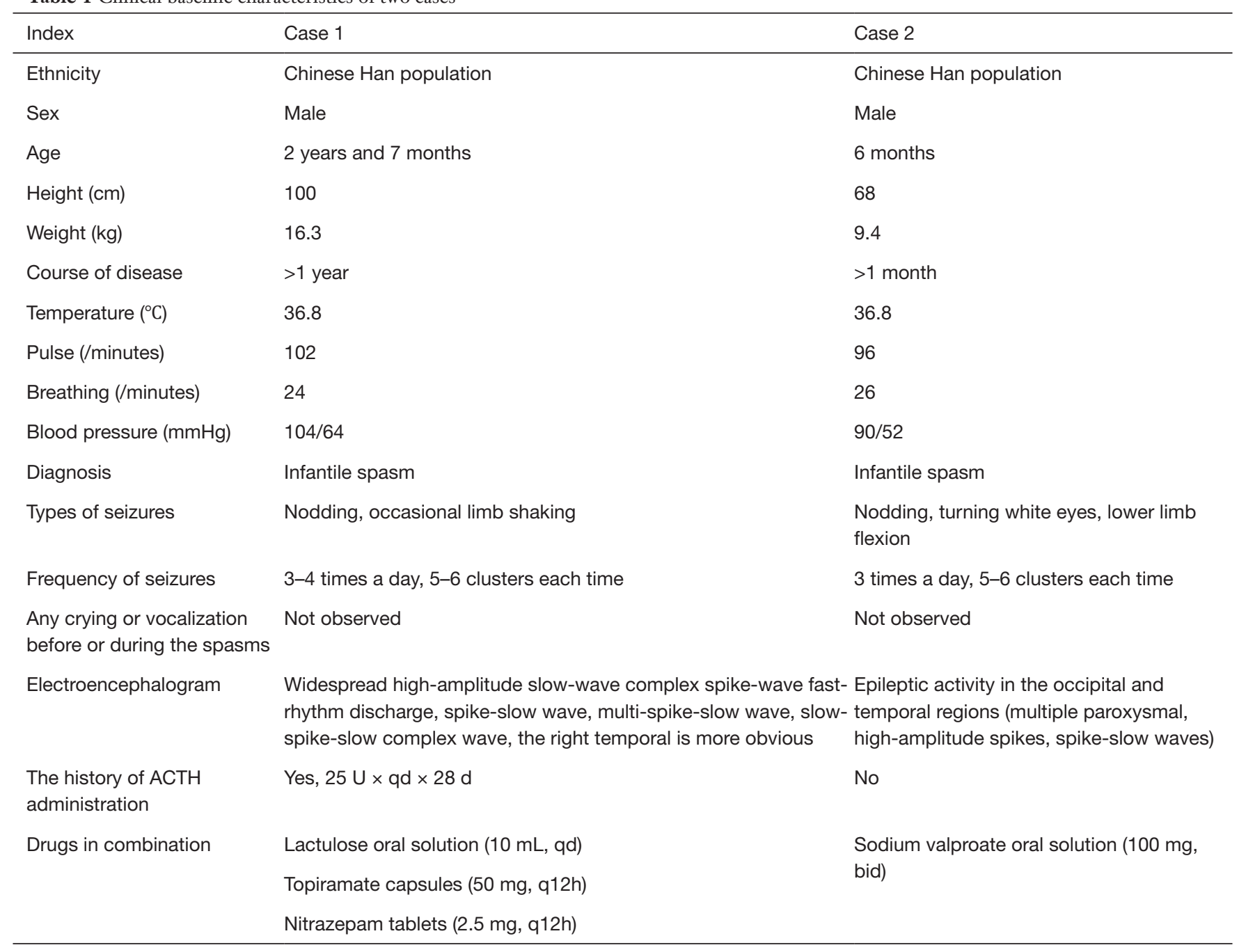

ACTH, adrenocorticotropic hormone.

could only be used during hospitalization because of its potential side effects, sodium valproate oral solution (2.5 mL, $100 \mathrm{mg}, 10.64 \mathrm{mg} / \mathrm{kg}$, bid) was used for epileptic treatment, but not significantly improved was observed after valproate treatment. This patient was hospitalized for further treatment of epileptic spasms on June $11^{\text {th }}, 2019$.

Clinical characteristics of this patient before ACTH therapy were shown in Table 1. The blood pressure of this patient was $90 / 52 \mathrm{mmHg}$ before ACTH therapy. Results of main laboratory test during ACTH therapy were also listed in Table 2. The ACTH treatment cycle and dosage of this patient were shown in Figure 1B. Interestingly, the blood pressure of this patient also increased to an abnormal range on the $9^{\text {th }}$ day $(102 / 68 \mathrm{mmHg})$. Although the ACTH therapy dose was adjusted from 40 to $25 \mathrm{U}$, the blood pressure of this patient reached to an abnormal high level range from $114 / 60 \mathrm{mmHg}$ in the morning to $122 / 78 \mathrm{mmHg}$ in the evening. This patient discontinued ACTH therapy on the $11^{\text {th }}$ day since ACTH therapy (Figure $1 B$ ). The blood pressure of this patient returned to normal range on the next day. There was no abnormal increase in blood pressure of this patient after discharge from hospital.

\section{Investigations}

Clinical investigations were performed to assess for the patient's treatment. They included patient's characteristic, results from laboratory tests, co-administrated drugs and 
Table 2 Results of main laboratory test

\begin{tabular}{|c|c|c|c|c|c|c|}
\hline Index (normal range) & \multicolumn{3}{|c|}{ Case 1} & \multicolumn{3}{|c|}{ Case 2} \\
\hline \multicolumn{7}{|l|}{ Regular blood analysis } \\
\hline White blood cell count $\left(4.0-15.0 \times 10^{9} / L\right)$ & 6.24 & $15.24 \uparrow$ & $15.94 \uparrow$ & 7.88 & 10.21 & / \\
\hline Red blood cell count $\left(3.70-5.80 \times 10^{12} / \mathrm{L}\right)$ & 4.61 & 4.5 & 4.87 & 4.45 & 4.51 & / \\
\hline Platelet count (100-550×109/L) & 281 & 369 & 348 & 370 & $629 \uparrow$ & / \\
\hline Neutrophil \% (50-70\%) & $26.6 \downarrow$ & $45.2 \downarrow$ & $42.3 \downarrow$ & $13.1 \downarrow$ & $42 \downarrow$ & / \\
\hline Neutrophil $\left(2.40-4.00 \times 10^{9} / L\right)$ & $1.66 \downarrow$ & $6.89 \uparrow$ & $6.74 \uparrow$ & $1.04 \downarrow$ & $4.29 \uparrow$ & / \\
\hline Basophil \% (0.00-0.75\%) & $1.4 \uparrow$ & 0.00 & 0.20 & 0.40 & 0.01 & / \\
\hline Lymphocyte (1.2-3.4×10\%/L) & $3.93 \uparrow$ & $6.88 \uparrow$ & $8.38 \uparrow$ & $5.95 \uparrow$ & $5.23 \uparrow$ & / \\
\hline Neutrophil to lymphocyte rate & 0.42 & 1.00 & 0.80 & 0.17 & 0.82 & \\
\hline \multicolumn{7}{|l|}{ Serum electrolyte level analysis } \\
\hline ECO2 (22-30 mmol/L) & $18 \downarrow$ & $21 \downarrow$ & / & 24 & $20 \downarrow$ & / \\
\hline $\mathrm{Na}^{+}(137-145 \mathrm{mmol} / \mathrm{L})$ & 143.8 & 141.4 & / & 140.1 & 137.0 & / \\
\hline $\mathrm{K}^{+}(3.5-5.1 \mathrm{mmol} / \mathrm{L})$ & 4.74 & 3.53 & / & 5.24 & 5.58 & / \\
\hline $\mathrm{Cl}^{-}(98-107 \mathrm{mmol} / \mathrm{L})$ & $110 \uparrow$ & $110 \uparrow$ & / & 104 & 100 & / \\
\hline \multicolumn{7}{|l|}{ Serum liver and kidney function analysis } \\
\hline Cystatin C (<1.09 mg/L) & 0.63 & 0.79 & / & 1.01 & 1.08 & \\
\hline Creatinine (9-88 $\mu \mathrm{mol} / \mathrm{L})$ & 25 & 23 & / & 24 & 21 & \\
\hline PreAlbumin $(0.09-0.12 \mathrm{~g} / \mathrm{L})$ & $0.2 \uparrow$ & $0.38 \uparrow$ & / & $0.34 \uparrow$ & $0.41 \uparrow$ & / \\
\hline \multicolumn{7}{|l|}{ Plasma lactic acid level analysis } \\
\hline Lac $(0.7-2.1 \mathrm{mmol} / \mathrm{L})$ & $2.57 \uparrow$ & $4.51 \uparrow$ & $2.57 \uparrow$ & 1.63 & / & / \\
\hline \multicolumn{7}{|l|}{ Serum cortisol function analysis } \\
\hline Cortisol (6.7-22.6 ㅆg/dL) & $4.9 \downarrow$ & / & / & 6.3 & / & / \\
\hline ACTH $(5.0-45.0 \mathrm{pg} / \mathrm{mL})$ & 8.63 & / & / & 21.3 & / & / \\
\hline \multicolumn{7}{|l|}{ Blood drug concentration monitoring } \\
\hline Serum valproic acid (50-100 mg/L) & / & / & / & / & / & $37 \downarrow$ \\
\hline
\end{tabular}

$\mathrm{B}$, before the ACTH administration; A, after discontinuation of ACTH; ACTH, adrenocorticotropic hormone; ALT, Alanine aminotransferase; AST, Aspartate aminotransferase; TP, total protein; TBIL, total bilirubin; Lac, lactic acid. 

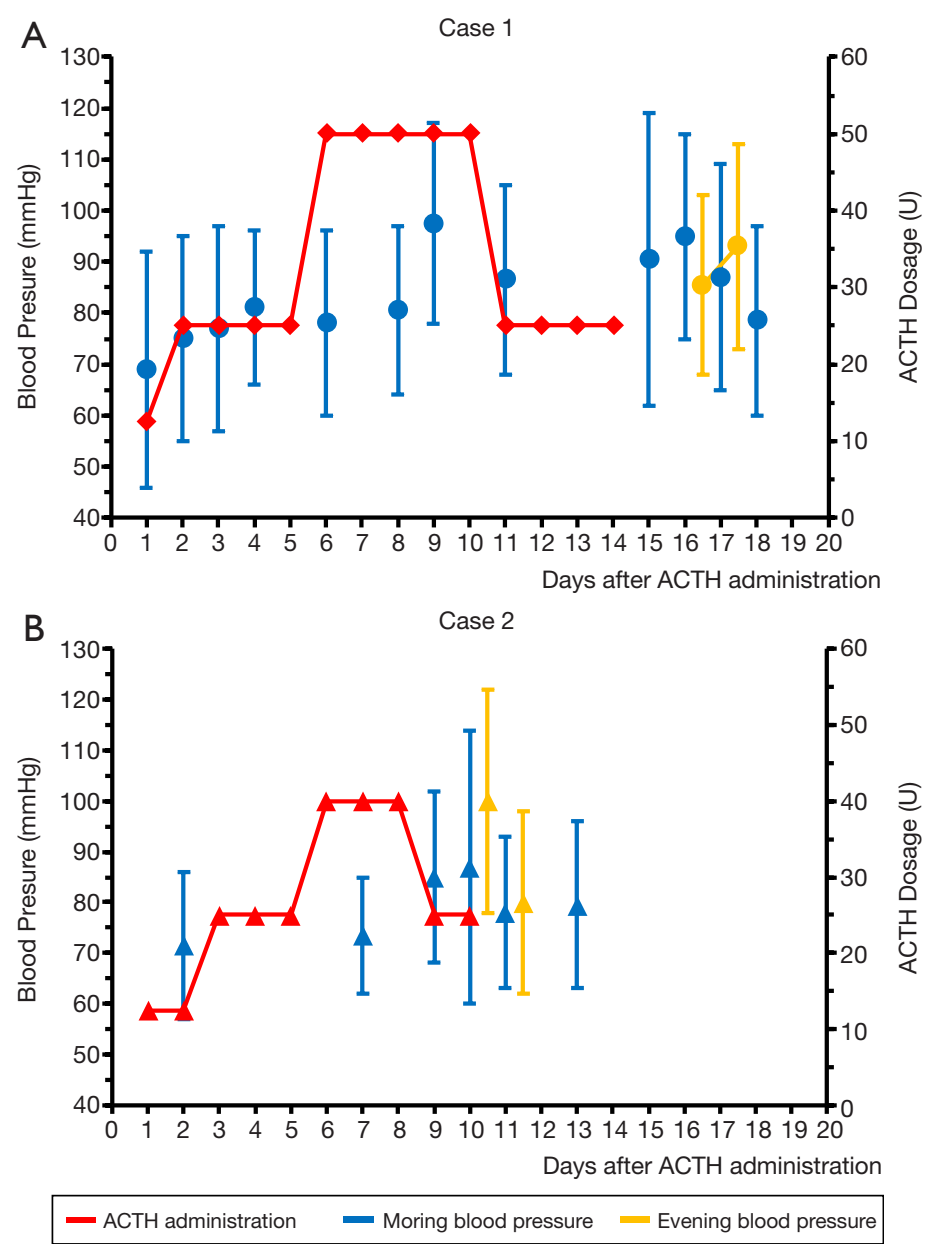

Figure 1 The relationship between ACTH therapy and blood pressure levels in two children with epileptic spasms. (A) Case 1; (B) Case 2 . ACTH, adrenocorticotropic hormone.

blood pressure levels.

\section{Laboratory tests}

Laboratory tests were completed in the laboratory of National Children's Medical Center, which included regular blood analysis, serum electrolyte level analysis, serum liver and kidney function analysis, plasma lactic acid level analysis, serum cortisol function analysis and blood drug concentration monitoring. Results of laboratory tests were listed in Table 2. Interestingly, both patients were associated with a lower level of neutrophil\%, neutrophil count, and a higher level of lymphocyte\%, lymphocyte and prealbumin than normal range before ACTH therapy. The white blood cell count level of the first patient (Case 1) was slightly higher than normal range.

\section{Drugs therapeutic program}

Two patients were treated with intravenous infusion of ACTH with an initial dose $12.5 \mathrm{U} / \mathrm{d}$. The therapeutic dosage of ACTH was gradually increased. All patients planned to receive ACTH therapy for 14 days. The maximum dose of ACTH for the first patient who was over 2 years old was $50 \mathrm{U}$. The second patient who was under 2 years old was treated with a maximum ACTH dose of 40 U. Detailed ACTH therapeutic program of 2 cases were shown in Figure 1.

\section{Blood pressure measurement and evaluation}

Blood pressure levels of all patients treated with ACTH were tested before and during ACTH therapy by using aneroid sphygmomanometer. Diagnosis and assessment 

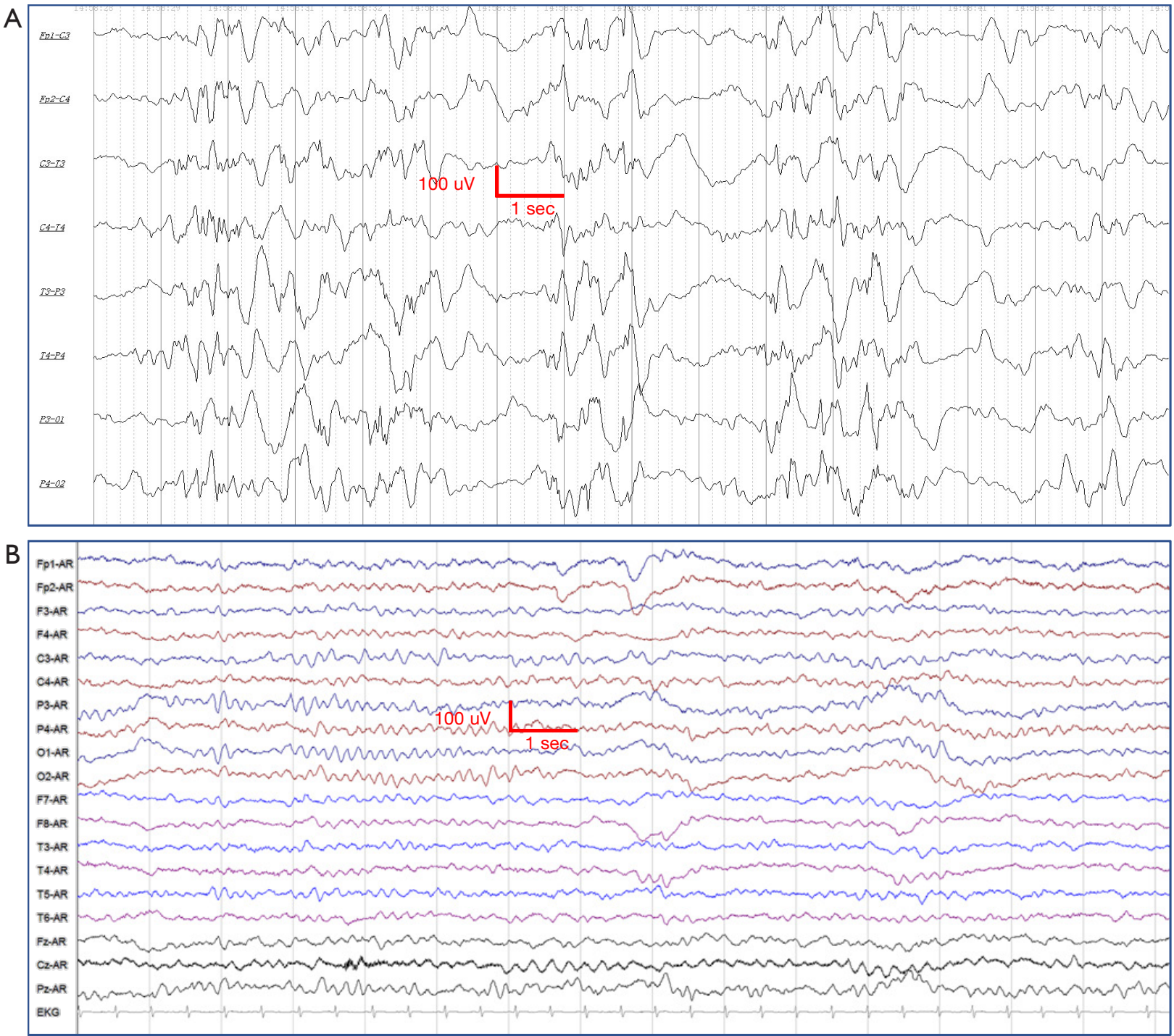

Figure 2 Classic EEG of case 2 consist of a high amplitude with multifocal spikes. (A) Before ACTH therapy, 8 channel ambulatory EEG of case 2 consisted with multiple paroxysmal, high-amplitude spikes, spike-slow waves. There was no well-formed anterior-posterior gradient or discernible posterior dominant rhythm; (B) after ACTH therapy, results from 16 channel digital audiovisual EEG of case 2 indicated that his EEG activity returned to normal range. EEG, electroencephalography; ACTH, adrenocorticotropic hormone.

of hypertension or abnormal high blood pressure in children were combining blood pressure varies with age, sex, and height. If blood pressure was $\geq 95^{\text {th }}$ percentile $\left(\mathrm{P}_{95}\right)$, we recorded it as an abnormal high blood pressure. Subsequently, the abnormal high blood pressure was staged according Hypertension Canada's 2018 Guidelines (14). Two stages were used to identify the severity of high blood pressure. If the blood pressure was between the $\mathrm{P}_{95}$ and $99^{\text {th }}$ percentile $\left(\mathrm{P}_{99}\right)$ plus $5 \mathrm{mmHg}$, the high blood pressure was defined as Stage 1 . If the blood pressure was $>\mathrm{P}_{99}$ plus
$5 \mathrm{mmHg}$, it was defined as stage 2 (14). The $\mathrm{P}_{95}$ data and $\mathrm{P}_{99}$ data of blood pressure of Chinese children aged from 3 to 17 years were obtained from results reported by Fan $e t$ al. $(15,16)$. Due to the lack of blood pressure data of Chinese children under 3 years old, we used data from 3-year-old children combined with individual characteristics of patients to determine the normal range of blood pressure of each patients. For 3-year-old patients with height under $96 \mathrm{~cm}$, the $\mathrm{P}_{95}$ and $\mathrm{P}_{99}$ systolic pressure were 102- and 108-mmHg, while the $\mathrm{P}_{95}$ and $\mathrm{P}_{99}$ diastolic pressure were 65 - and 
72-mmHg, respectively. For 3-year-old patients with height between 98 to $100 \mathrm{~cm}$, the $\mathrm{P}_{95}$ and $\mathrm{P}_{99}$ systolic pressure were 104- and $110-\mathrm{mmHg}$, while the $\mathrm{P}_{95}$ and $\mathrm{P}_{99}$ diastolic pressure were $66-$ and $72-\mathrm{mmHg}$, respectively. For case 1 , the blood pressure of the $9^{\text {th }}, 15^{\text {th }}$ and $16^{\text {th }}$ day after ACTH therapy reached high blood pressure stage 2 (systolic pressure $>115 \mathrm{mmHg}$ ). And the blood pressure of case 2 reached high blood pressure stage 2 on the $10^{\text {th }}$ day after ACTH therapy.

\section{Discussion}

ACTH was approved by the US Food and Drug Administration for use in patients since 1952 (17). It could stimulate the ACTH receptor, which lead to a stimulation of the adrenal cortex, resulting in the increase of cortisol production. In 2004, a report from American Academy of Neurology/Child Neurology Society indicated that ACTH was an effective agent for the treatment of epileptic spasms $(18,19)$. The cost for epileptic spasms treatment with ACTH estimated about US\$70,000 to US\$150,000 in the USA, and about RMB5,000 (US\$715) for a 14 days course of ACTH therapy in China $(3,9)$. Oral corticosteroids are another choice for epileptic spasms therapy. Synthesizing glucocorticoid made corticosteroids oral administration available and a drop-in price (17). But oral corticosteroids are used off-label for the treatment of epileptic spasms (3). Although the Unite States Food and Drug Administration approve ACTH for the treatment of infantile spasms in infants and children under 2 years of age, ACTH are still used off-label for the treatment of epileptic spasms in China. Patients with epileptic spasms resulted from etiologies but not tuberous sclerosis complex were still recommended ACTH as first line intervention (19). Results from Chinese experts Yin et al. indicated that $14^{\text {th }}$ day of ACTH treatment could be a useful time point to evaluate whether such patient need a continual ACTH treatment for up to 28 days (20). They also found that maintaining the same ACTH dose resulted in a higher response rate than doubling the ACTH dose in patients with infantile spasms (20). Results from our previous study indicated that short-term (14 days) of the ACTH incremental therapy was also effective for the treatment of infantile spasms with a low incidence of adverse reaction (21). For the first patient (Case 1), he received a 28 days of ACTH treatment previously in Soochow. But for both patients, they were treated with 14 days ACTH incremental therapy in our hospital. After ACTH treatment, oral corticosteroids such as prednisone are considered as follow up treatment instead of gradually tapered and discontinued of a 2 -week period ACTH therapy.

The chief risks of ACTH therapy are immunosuppression and hypertension as other hormonal therapies (12). Immunosuppression, which is always associated with infections, can be severe and lethal. In our cases report, the white blood cell counts of the first patient (Case 1) were slightly higher than normal range raised to $15.24 \times 10^{9} / \mathrm{L}$ on the $11^{\text {th }}$ day since ACTH therapy and $15.94 \times 10^{9} / \mathrm{L}$ at the end of ACTH therapy, which indicated that infection might accompanied with this patient. But highly blood pressures were observed in both patients during the ACTH therapy in our study. Persistent hypertension of children may lead to yield congestive heart failure $(13,22)$. Children can be diagnosed as hypertensive when the blood pressure measured on at least 3 separate occasions were $\geq \mathrm{P}_{95}$ data for age, sex and height (14). In our cases report, both patients were reached high blood pressure stage 2, which indicated that the blood pressure of both patients were extremely high. Due to the lack of standards of blood pressure for children under 3 years old, we can only estimate the standard ranges for children under 3 years old by their sex and closest height. The standard ranges of blood pressure increase with age and height (15). This suggests that the highly blood pressure in these two children may be more serious. Our previous study showed that the incidence of high blood pressure of patients who suffered from infantile spasms with incremental ACTH therapy was 2.5\% (21). Results from Yin et al. indicated that after different once-daily doses of ACTH for Chinese patients with infantile spasms, the incidences of high blood pressure were ranged from $13.5 \%$ to $23.6 \%$ (20). However, Eliyan et al. pointed out that hypertension was not more common among infantile spasms patients who treated with ACTH and prednisolone when compared with prednisolone monotherapy (23). Results from Angappan et al. found after intramuscular ACTH in infants with West syndrome in India, hypertension was the predominant adverse effect with an incidence of $93.3 \%$ (24). Recently, a retrospective study reported by McGarry et al. quantified the incidence of hypertension and indicated that hypertension occurred in $44 \%$ children who suffered from infantile spasms during the treatment with ACTH (25). Although the incidence of hypertension among patients treated with ACTH is subject to ongoing debate, there is still needing to screen for asymptomatic high blood pressure before and during ACTH therapy.

In this cases report, both patients were found with high blood pressure stage 2 on the $9^{\text {th }}$ day and $10^{\text {th }}$ day of ACTH treatment. Interestingly, the lower level of neutrophil\%, 
neutrophil count, and a higher level of lymphocyte\%, lymphocyte count and prealbumin than normal range were found in both patients before starting ACTH therapy. Neutrophil to lymphocyte rate might be a predictor for incident hypertension. Results from a 9-year cohort study in Taiwan proved this hypothesis (26). It was also demonstrated in a large-scale cohort (27). Neutrophils were associated with inflammatory processes and were involved in releasing reactive oxygen species, which were involved in nitric oxide-based cell signaling resulted in the vascular disorders potentially associated with hypertension (28,29). Lymphocyte, especially T lymphocyte, also participate in the hypertensive process. Limitation of CD28 and B7 ligation in T lymphocyte resulted in the attenuated of hypertensive condition (30). Furthermore, stimulation of $\mathrm{T}$ lymphocytes also resulted in angiotensin II -mediated hypertensive response (31). During the ACTH therapy, the fast increasing the level of neutrophil but not lymphocyte resulted in the abnormal high blood pressure. We think neutrophil to lymphocyte rate before starting ACTH therapy can be used as a predictor for incident hypertension. But all the related evidences were obtained from adults' patients, especially in elderly groups $(26,27)$. The normal range of neutrophil to lymphocyte rate among these patients is between 0.78 and 3.53 (32). In this cases report, the neutrophil to lymphocyte rates of both patients before ACTH therapy were 0.42 and 0.17 respectively. We deduced neutrophil to lymphocyte rate lower than 0.5 before starting ACTH therapy could predict high blood pressure among these patients who suffered from epileptic spasms and treated with ACTH. Whether these patients are already predisposed for incidental hypertension and the ACTH treatment pushes them over the edge is needed further evaluation in a larger study in the future.

Serum prealbumin (also as known as transthyretin), is a serum protein secreted mainly by the liver with short half-life. It is mainly involved in thyroid hormones transportation (33). Prealbumin can be used as a predictor of survival in hemodialysis and peritoneal dialysis (34). End-stage renal disease patients with prealbumin greater than $0.3 \mathrm{~g} / \mathrm{L}$ were associated with a significantly higher survival rate (34). For patients receiving hemodialysis, lower serum prealbumin were also associated with a higher death risk (35). Results from Chrysostomou et al. indicated hemodialysis patients had higher levels of prealbumin when compared with renal-transplant patients (36). More and more results indicated serum prealbumin is potent predictor for not only nutritional evaluation, but also for disease prognosis evaluation $(37,38)$. However, the relation between serum prealbumin and blood pressure is still unknown. Whether ACTH treatment would induce a high level of serum prealbumin, which might increase serum albumin level and affect plasma colloid osmotic pressure, lead to water retention result in abnormal high blood pressure is also unknown. There is nothing direct evidence to explain the relationship between serum prealbumin and hypertension. We assumed that the high level of prealbumin leaded to an increase of thyroid hormones transport, which resulted in the increase of blood pressure (39).

When predicting one patient may associated with abnormal high blood pressure during ACTH therapy, the most important thing is how to prevent or how to deal with it. A randomized, open-label clinical trial by Professor Li-Ping Zou et al. indicated that ACTH combination treatment with magnesium sulfate $\left(\mathrm{MgSO}_{4}\right)$ for infantile spasms resulted in zero case of hypertension, insomnia and irritability (40). Furthermore, they also found that $\mathrm{MgSO}_{4}$ had a neuroprotective effect (40). Deficiency of magnesium resulted in high arterial blood pressure and spasms of vessels $(41-45)$. It is also associated with high blood pressure in children (45). $\mathrm{MgSO}_{4}$ could dilate the constricted muscles in the arteries, which can be used for blood pressure control, with an acceptable safety for infants $(46,47)$.

The strength of our report was to document that the neutrophil to lymphocyte rate might predict high blood pressure among epileptic spasms patients treated with ACTH. But our report also had a few potential limitations. Firstly, our study was underpowered to detect the high blood pressure and epilepsy related genes. Secondly, the serum prealbumin as a predictor for high blood pressure was only theoretical speculation, there was no direct evidence of the relationship between prealbumin and blood pressure. Moreover, the normal range of blood pressure for children under 3 years old was speculated according to data from 3-year-old children.

\section{Conclusions}

Our results indicated that the neutrophil to lymphocyte rate and serum prealbumin might predictors for high blood pressure in epileptic spasms patients treated with ACTH.

\section{Acknowledgments}

Funding: This work was supported by the Fundamental Research Funds for the Center Universities (No. 
17JCYB11) and Shanghai Municipal Hospital new frontier technology project (SHDC 12015113).

\section{Footnote}

Conflicts of Interest: The authors have no conflicts of interest to declare.

Ethical Statement: The authors are accountable for all aspects of the work in ensuring that questions related to the accuracy or integrity of any part of the work are appropriately investigated and resolved. Written informed consent was obtained from the patients for publication of this manuscript.

Open Access Statement: This is an Open Access article distributed in accordance with the Creative Commons Attribution-NonCommercial-NoDerivs 4.0 International License (CC BY-NC-ND 4.0), which permits the noncommercial replication and distribution of the article with the strict proviso that no changes or edits are made and the original work is properly cited (including links to both the formal publication through the relevant DOI and the license). See: https://creativecommons.org/licenses/by-nc-nd/4.0/.

\section{References}

1. Wilmshurst JM, Ibekwe RC, O'Callaghan FJK. Epileptic spasms - 175 years on: Trying to teach an old dog new tricks. Seizure 2017;44:81-6.

2. Fisher RS. The New Classification of Seizures by the International League Against Epilepsy 2017. Curr Neurol Neurosci Rep 2017;17:48.

3. Raga SV, Wilmshurst JM. Epileptic spasms: Evidence for oral corticosteroids and implications for low and middle income countries. Seizure 2018;59:90-8.

4. Berg AT, Berkovic SF, Brodie MJ, et al. Revised terminology and concepts for organization of seizures and epilepsies: report of the ILAE Commission on Classification and Terminology, 2005-2009. Epilepsia 2010;51:676-85.

5. Zupanc ML. Infantile spasms. Expert Opin Pharmacother 2003;4:2039-48.

6. Infantile Spasms of Unknown Cause: Who Can Have a Good Outcome? Epilepsy Curr 2019;19:171-3.

7. Osborne JP, Lux AL, Edwards SW, et al. The underlying etiology of infantile spasms (West syndrome): information from the United Kingdom Infantile Spasms Study (UKISS) on contemporary causes and their classification. Epilepsia 2010;51:2168-74.

8. Knupp KG, Coryell J, Nickels KC, et al. Response to treatment in a prospective national infantile spasms cohort. Ann Neurol 2016;79:475-84.

9. Lux AL. Latest American and European updates on infantile spasms. Curr Neurol Neurosci Rep 2013;13:334.

10. Nelson GR. Management of infantile spasms. Transl Pediatr 2015;4:260-70.

11. Kelley SA, Knupp KG. Infantile Spasms-Have We Made Progress? Curr Neurol Neurosci Rep 2018;18:27.

12. Hussain SA. Treatment of infantile spasms. Epilepsia Open 2018;3:143-54.

13. Riikonen R, Donner M. ACTH therapy in infantile spasms: side effects. Arch Dis Child 1980;55:664-72.

14. Nerenberg KA, Zarnke KB, Leung AA, et al. Hypertension Canada's 2018 Guidelines for Diagnosis, Risk Assessment, Prevention, and Treatment of Hypertension in Adults and Children. Can J Cardiol 2018;34:506-25.

15. Fan H, Yan Ya, Mi J. Updating blood pressure references for Chinese children aged 3-17 years. Chinese Journal of Hypertension 2017;25:428-35.

16. Fan H, Yan YK, Mi J. Establishing the user-friendly screening criteria for elevated blood pressure in Chinese children aged 3-17 years. Chinese Journal of Hypertension 2017;25:436-40.

17. Montero-Melendez T. ACTH: The forgotten therapy. Semin Immunol 2015;27:216-26.

18. Mackay MT, Weiss SK, Adams-Webber T, et al. Practice parameter: medical treatment of infantile spasms: report of the American Academy of Neurology and the Child Neurology Society. Neurology 2004;62:1668-81.

19. Go CY, Mackay MT, Weiss SK, et al. Evidence-based guideline update: medical treatment of infantile spasms. Report of the Guideline Development Subcommittee of the American Academy of Neurology and the Practice Committee of the Child Neurology Society. Neurology 2012;78:1974-80.

20. Yin J, Lu Q, Yin F, et al. Effectiveness and Safety of Different Once-Daily Doses of Adrenocorticotropic Hormone for Infantile Spasms. Paediatr Drugs 2017;19:357-65.

21. Dai S, He Y, Chen Y, et al. Clinical study on treatment of infantile spasms with incremental corticotrophin therapy. Chinese Journal of Applied Clinical Pediatrics 2019;34:513-6.

22. Battistoni A, Canichella F, Pignatelli G, et al. Hypertension in Young People: Epidemiology, Diagnostic Assessment and Therapeutic Approach. High Blood Press Cardiovasc 
Prev 2015;22:381-8.

23. Eliyan Y, Heesch J, Alayari A, et al. Very-High-Dose Prednisolone Before ACTH for Treatment of Infantile Spasms: Evaluation of a Standardized Protocol. Pediatr Neurol 2019;99:16-22.

24. Angappan D, Sahu JK, Malhi P, et al. Safety, tolerability, and effectiveness of oraal zonisamide therapy in comparison with intramuscular adrenocorticotropic hormone therapy in infants with West syndrome. Eur J Paediatr Neurol 2019;23:136-42.

25. McGarry L, Messer R, Cree-Green M, et al. Incidence of Hypertension Among Children Treated With Adrenocorticotropic Hormone (ACTH) or Prednisolone for Infantile Spasms. J Child Neurol 2020;35:215-20.

26. Jhuang YH, Kao TW, Peng TC, et al. Neutrophil to lymphocyte ratio as predictor for incident hypertension: a 9-year cohort study in Taiwan. Hypertens Res 2019;42:1209-14.

27. Liu X, Zhang Q, Wu H, et al. Blood Neutrophil to Lymphocyte Ratio as a Predictor of Hypertension. Am J Hypertens 2015;28:1339-46.

28. Vallance P, Collier J, Bhagat K. Infection, inflammation, and infarction: does acute endothelial dysfunction provide a link? Lancet 1997;349:1391-2.

29. Pierini D, Bryan NS. Nitric oxide availability as a marker of oxidative stress. Methods Mol Biol 2015;1208:63-71.

30. Vinh A, Chen W, Blinder Y, et al. Inhibition and genetic ablation of the B7/CD28 T-cell costimulation axis prevents experimental hypertension. Circulation 2010;122:2529-37.

31. Crowley SD, Frey CW, Gould SK, et al. Stimulation of lymphocyte responses by angiotensin II promotes kidney injury in hypertension. Am J Physiol Renal Physiol 2008;295:F515-24.

32. Forget P, Khalifa C, Defour JP, et al. What is the normal value of the neutrophil-to-lymphocyte ratio? BMC Res Notes 2017;10:12.

33. Ingenbleek Y, Young V. Transthyretin (prealbumin) in health and disease: nutritional implications. Annu Rev Nutr 1994;14:495-533.

34. Sreedhara R, Avram MM, Blanco M, et al. Prealbumin is the best nutritional predictor of survival in hemodialysis and peritoneal dialysis. Am J Kidney Dis 1996;28:937-42.

35. Rambod M, Kovesdy CP, Bross R, et al. Association of serum prealbumin and its changes over time with clinical outcomes and survival in patients receiving hemodialysis. Am J Clin Nutr 2008;88:1485-94.

36. Chrysostomou S, Stathakis C, Petrikkos G, et al. Assessment of prealbumin in hemodialysis and renal- transplant patients. J Ren Nutr 2010;20:44-51.

37. Loftus TJ, Brown MP, Slish JH, et al. Serum Levels of Prealbumin and Albumin for Preoperative Risk Stratification. Nutr Clin Pract 2019;34:340-8.

38. Beck FK, Rosenthal TC. Prealbumin: a marker for nutritional evaluation. Am Fam Physician 2002;65:1575-8.

39. Power DM, Elias NP, Richardson SJ, et al. Evolution of the thyroid hormone-binding protein, transthyretin. Gen Comp Endocrinol 2000;119:241-55.

40. Zou LP, Wang X, Dong CH, et al. Three-week combination treatment with ACTH + magnesium sulfate versus ACTH monotherapy for infantile spasms: a 24week, randomized, open-label, follow-up study in China. Clin Ther 2010;32:692-700.

41. Altura BM, Altura BT, Gebrewold A, et al. Magnesium deficiency and hypertension: correlation between magnesium-deficient diets and microcirculatory changes in situ. Science 1984;223:1315-7.

42. Altura BM, Altura BT, Carella A. Magnesium deficiencyinduced spasms of umbilical vessels: relation to preeclampsia, hypertension, growth retardation. Science 1983;221:376-8.

43. Van Laecke S, Vanholder R. Magnesium and vascular dysfunction in malignant hypertension. Hypertension 2011;58:e7; author reply e8.

44. Cunha AR, Umbelino B, Correia ML, et al. Magnesium and vascular changes in hypertension. Int J Hypertens 2012;2012:754250.

45. Guerrero-Romero F, Rodriguez-Moran M, HernandezRonquillo G, et al. Low Serum Magnesium Levels and Its Association with High Blood Pressure in Children. J Pediatr 2016;168:93-8.e1.

46. Kostov K, Halacheva L. Role of Magnesium Deficiency in Promoting Atherosclerosis, Endothelial Dysfunction, and Arterial Stiffening as Risk Factors for Hypertension. Int J Mol Sci 2018. doi: 10.3390/ijms19061724.

47. Zeng X, Xue Y, Tian Q, et al. Effects and Safety of Magnesium Sulfate on Neuroprotection: A Meta-analysis Based on PRISMA Guidelines. Medicine (Baltimore) 2016;95:e2451.

Cite this article as: $\mathrm{Li} \mathrm{H,} \mathrm{Wang} \mathrm{CJ,} \mathrm{Zhou} \mathrm{YQ,} \mathrm{Wang} \mathrm{YY,}$ Mou CH, Zhang SG, Wang JW. Neutrophil to lymphocyte rate and serum prealbumin maybe predictors for abnormal high blood pressure caused by adrenocorticotropic hormone therapy in children with epileptic spasms: two cases report. Ann Transl Med 2020;8(5):248. doi: 10.21037/atm.2020.01.132 